\title{
Genotypic-dependent amphetamine effects in rats bred for differences in alcohol sensitivity
}

\author{
NEIL R. SHAPIRO, AJAY P. GARG, and EDWARD P. RILEY \\ State University of New York, Albany, New York 12222
}

\begin{abstract}
Locomotor activity in an open field was assessed in two selectively bred rat lines following an IP injection of $0,1.5$, or $3.0 \mathrm{mg} / \mathrm{kg} \mathrm{d}$-amphetamine. The MA rats, bred for alcohol sensitivity, evidenced less of an increase in ambulation following amphetamine than did LA rats, bred for alcohol insensitivity. Linear dose-response functions with disparate slopes were found for the MA and LA rats, with activity of these lines becoming more divergent as dosage increased. Amphetamine increased rearing frequency in both lines, and though it appeared that the LA line evidenced greater activation at the intermediate dose, this was not statistically significant. Amphetamine was also found to produce a dose-dependent decrease in the duration of each rear, and this function appeared similar for the two lines. These data were compared to reports that inbred strains of mice which differ in sleep time following alcohol challenge also are distinguished in response to amphetamine.
\end{abstract}

Two lines of rats have been selectively bred by Riley, Freed, and Lester (1976) for differences in motor impairment following alcohol challenge. The "most" affected (MA) line shows approximately a $90 \%$ reduction in gross motor activity following the standard ethanol dose used in selection, $1.5 \mathrm{mg} / \mathrm{kg}$, compared to only a $40 \%$ reduction in the "least", affected (LA) line (Worsham, Riley, Anandam, Lister, Freed, \& Lester, 1977). Furthermore, the disparate impairment of these lines appears consistent across several subhypnotic (Worsham \& Freed, 1977) and hypnotic doses of alcohol (Riley \& Lochry, 1977; Riley, Shapiro, \& Lochry, 1979; Riley, Worsham, Lester, \& Freed, 1977). In addition to generality across dose, the differential sensitivity of these lines to alcohol is evident with several other spontaneous activity measures, including open-field ambulation and wheel running (Worsham, Freed, Lochry, \& Riley, 1977). More recently, it has been shown that MA rats also evidence significantly greater impairment of a conditioned avoidance response following alcohol challenge, extending the findings of alcoholsensitivity differences in these lines to an elicited response (Shapiro \& Riley, Note 1).

It has been suggested that the disparate response of the lines to alcohol represents a difference in neural sensitivity to this drug, since the two lines clear alcohol at similar rates (Lester, Lin, Anandam, Riley, Worsham, \& Freed, 1977) and do not appear to differentially absorb it (Worsham, Riley et al.,

Requests for reprints should be sent to Edward P. Riley, Department of Psychology, State University of New York at Albany, Albany, New York 12222. We would like to thank Dr. Ralph Myerson of Smith, Kline, and French Labs, Philadelphia, for the gift of d-amphetamine.
1977). Moreover, it appears that the lines may differ along a more general phenotype than previously believed, since the lines can also be distinguished by their response to other depressants, including two different barbiturates, pentobarbital and phenobarbital, as well as the nonbarbiturate hypnotic, chloral hydrate (Riley, Lochry, \& Freed, 1978; Riley et al., 1979).

Since drug manipulations with these lines had not extended beyond those having a depressant action, the following experiment was conducted in order to assess whether the lines would evidence differential response increments following the administration of a stimulant. Open-field ambulation and rearing were measured for MA and LA rats following adminstration of d-amphetamine, a centrally acting stimulant shown to modify both of these responses (e.g., Fog, 1970).

\section{METHOD}

\section{Subjects}

Twenty-four naive rats between 75 and 100 days of age were randomly chosen from each line of the F19 generation, bred at SUNY-Albany. Details of the breeding procedure are described elsewhere (Riley et al., 1976). The animals were housed two per cage under a 12-h light cycle, with food and water coninuously available except as noted below. The subjects were equally divided between groups; however, one rat from the MA line scheduled to receive the high amphetamine dose was excluded from the experiment due to illness.

\footnotetext{
Apparatus

A square, Plexiglas open field, $63.5 \times 63.5 \times 30.5 \mathrm{~cm}$, was used. The apparatus was painted flat white on all surfaces except the lid, which was left transparent so that the animal could be observed. The open field was divided into 16 equal squares by intersecting infrared photobeams. Illumination was provided by a $6-\mathrm{W}$ red light centered above the apparatus.
} 


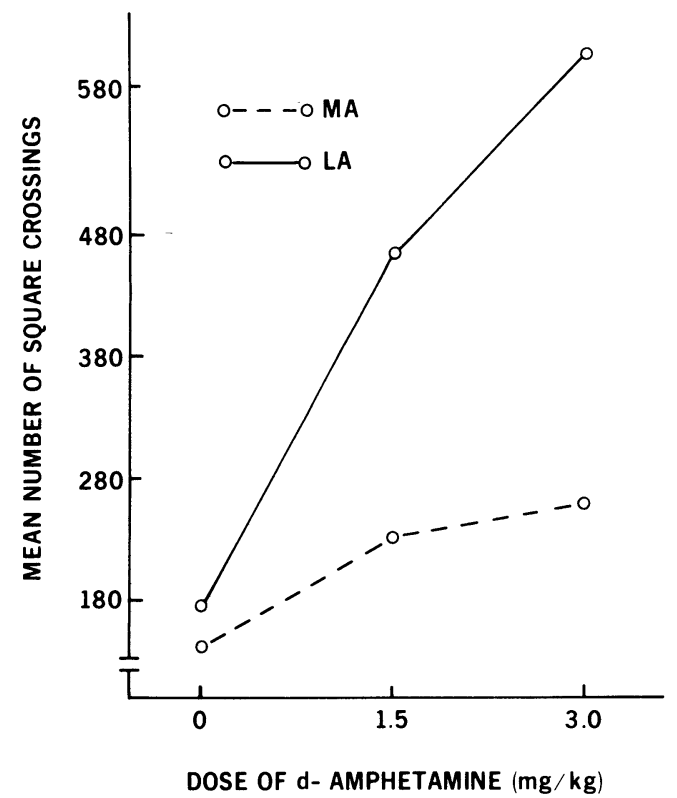

Figure 1. Mean number of square crossings by MA and LA rats as a function of amphetamine dose.

\section{Procedure}

Isotonic saline or d-amphetamine sulfate (Smith, Kline, \& French) at a dose of 1.5 or $3.0 \mathrm{mg} / \mathrm{kg}$ was administered to $24-\mathrm{h}$ food-deprived animals $15 \mathrm{~min}$ prior to testing. The amphetamine was prepared in a saline vehicle at a concentration of $3 \mathrm{mg} / \mathrm{ml}$, and thus the two amphetamine groups received different injection volumes. The injection volume of the saline group was equivalent, on a milliliter per kilogram basis, to that received by the higher dosage amphetamine group.

Rats from each line were placed individually in the center of the field, and consecutive beam crossings were automatically recorded for $15 \mathrm{~min}$. A count was registered each time the animal crossed a beam other than the one just previously broken. This measure roughly approximates the number of square crossings, except that it excludes retracing into the previous square.

The frequency and duration of rearing in the open field were assessed by one of two trained observers equipped with a silent pushbutton and positioned in such a way as to minimize distractions to the animal. The observers were unaware of the subject's genotype and dosage. Each time the animal lifted both forepaws off the floor of the open field without grooming, the observer pressed and held the pushbutton for the duration of the rear. Rearing frequency, duration to the 10th of a second, and the ambulation measure were recorded by solid state printers in an adjacent room.

\section{RESULTS}

The mean number of square crossings for each line as a function of amphetamine dose is presented in Figure 1. The marked difference in slope of the twodose-response functions suggests that LA rats, showing the greater increase, are more activated in terms of ambulation following this stimulant. It is also important to note that there appears to be little, if any, difference in activity of the lines following control injections.

An analysis of variance performed on these data with line and dose as between-subject factors revealed a significant interaction $[F(2,41)=5.16, p<.025]$, confirming the apparent difference in dose-response plots for the two lines. Breakdown of the interaction term into simple main effects (Winer, 1971, p. 444) revealed a significant linear effect of dose for both the MA $[F(1,20)=7.17, p<.025]$ and LA lines $[F(1,20)=23.38, p<.001]$. Thus, it appears that both MA and LA rats evidenced increased activity following amphetamine; however, the degree to which activity increased was dependent upon the animal's genotype.

The mean frequency of rearing and the duration per rear, derived by dividing the total duration by the frequency of rearing in that session, are presented for each line and dose in Panels A and B of Figure 2. As can be seen in the figure, amphetamine increased the number of rears made by animals of each line, while reducing the average duration of individual rearing response. Furthermore, an interaction of dose and line appears to be evident in terms of the frequency of rearing measure, since the two lines appear equivalent at the zero dose, but markedly differ at the $1.5-\mathrm{mg} / \mathrm{kg}$ amphetamine dosage. However, analyses of variance performed on the two rearing measures, with line, amphetamine dose, and observer as between-subject factors revealed only main effects for either dependent measure. Significant dose effects were revealed for both measures $[\mathrm{Fs}(2,35)=11.95$ and 3.30 , ps $<.001$ and .05 , for rearing frequency and duration per rear, respectively]. Overall doseresponse functions for each measure were linear; amphetamine produced a dose-dependent increase in rearing frequency and a dose-dependent decrease in duration per rear (Figure 2), as indicated by significant linear trend components for these two measures $[\mathrm{Fs}(1,35)=20.68$ and 6.60 , ps $<.001$ and .025 , re-

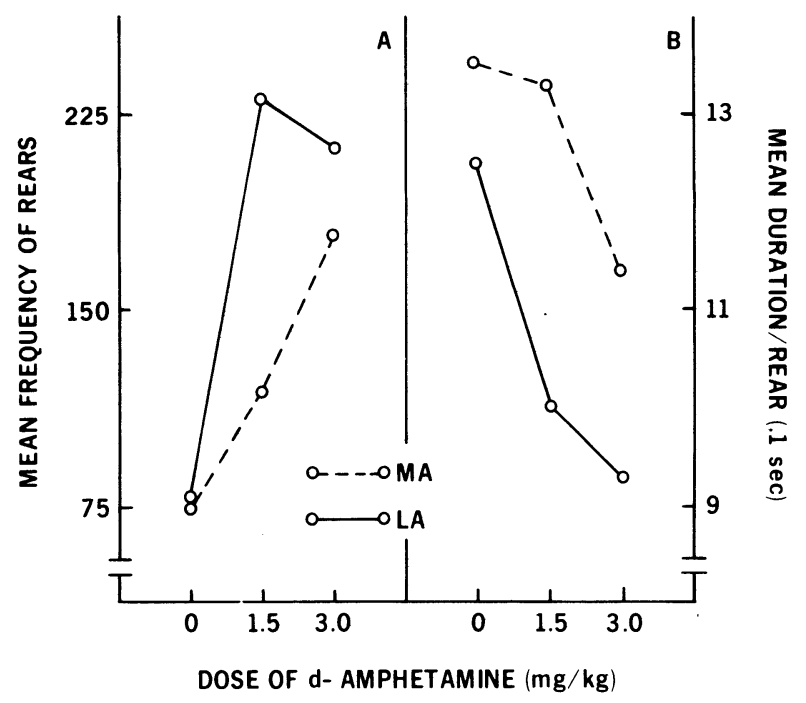

Figure 2. Mean frequency of rearing (Panel $A$ ) and mean duration of each rear in 10ths of seconds (Panel B) for MA and LA rats as a function of amphetamine dose. 
spectively] and by the absence of significant quadratic terms $[\mathrm{F}(1,35)=3.23, \mathrm{p}>.05$, and $\mathrm{F}<1]$. In addition, the effect of line was significant for both dependent measures $[F(1,35)=5.64, p<.025$, for rearing frequency and $F(1,35)=7.25, p<.025$, for duration per rear]. As noted, in neither case did the interaction of line and dose reach significance; however, there was a marginally nonsignificant Line by Dose-Quadratic interaction component for the rearing frequency measure $[\mathrm{F}(1,35)=4.10, \mathrm{p}=.051]$, providing some suggestion of a difference between the lines at the intermediate dose (see Figure 2). Reliability of observational recordings of rearing behavior within each condition is suggested by the absence of significant observer effects for rearing frequency or duration per rear, and by the absence of any interaction of this variable with line or dose for either dependent measure.

\section{DISCUSSION}

The present results indicate that LA rats show greater motor activation than rats of the MA line following administration of d-amphetamine. Furthermore, dose-response functions derived for this measure appear to indicate that this disparity in response of the lines increases with the dose administered. It is important to note that, in accord with previous findings (Riley et al., 1977), the lines did not differ in basal activity rates, mean activity being almost identical for the two saline control groups in the present experiment. Thus, the present findings appear to reflect genotypic-dependent stimulation of activity by amphetamine, independent of possible rate-dependent effects.

The effect of genotype was less evident with regard to amphetamine action on rearing behavior. Although the LA line appeared to evidence a greater increase in frequency of rearing following amphetamine, this difference was not statistically reliable. While increasing the frequency of rearing in both lines, d-amphetamine was found to produce a dose-dependent decrease in the duration of each rear, and this function also did not differ between lines. The reason for the discrepancy in results between rearing and ambulatory behaviors in the open field is not clear. However, it is possible that the response to amphetamine in terms of these two behaviors may be controlled by separate genes (Moisset, 1977).

The present activity differences following amphetamine treatment in MA and LA rats appears consistent with findings of strain differences in response to amphetamine among inbred mice which differ in loss of righting reflex durations (sleep time) following alcohol administration. For example, mice of the C57BL strain evidence shorter sleep times than BALB mice following alcohol (Damjanovich \& MacInnes, 1973; Kakihana, Brown, McClearn, \& Tabershaw, 1966), and in addition show a marked increase in open-field activity following amphetamine, whereas the BALB mice show little if any increase (Moisset \& Welch, 1973). Moreover, compared to C57BL mice, DBA mice show prolonged sleep times following alcohol administration (Damjanovich \& MacInnes, 1973), and evidence only small increases in activity following amphetamine (Meier, Hatfield, \& Foshee, 1963).

It might be noted that the behavior of the MA and LA lines appears to mirror that of the mouse strains in several other respects. Recall that the greater sensitivity to alcohol of MA rats compared to LA rats appears to generalize to other depressant drugs (Riley et al., 1978, 1979); likewise, the DBA and BALB mice, which show prolonged sleep times following alcohol, appear to evidence greater sleep times and motor incoordination than C57BL mice following other depressants (Belknap, Waddingham, \& Ondrusek, 1973; Meier et al., 1963). Additionally, parallels have been drawn in terms of two-way avoidance and running-wheel behaviors (Shapiro \& Riley, Note 1). However, it should be pointed out that the reactions of the mouse strains to alcohol and that of the MA and LA rats are not entirely analogous, in that responsiveness of these mouse strains to this drug may vary across dose, and the elimination of alcohol may occur at different rates (Damjanovich \& MacInnes, 1973).

In conjunction with the data concerning the inbred mice, the present findings suggest a relationship between the genetically determined effects of alcohol and amphetamine. It is premature, however, to speculate on the mechanism underlying the differential response of these lines. It is possible that the disparate activation following amphetamine may simply reflect differences in metabolism of this drug, since data concerning absorption and elimination rates were not collected. However, as there are no differences in the rate of alcohol clearance between these lines, it seems unlikely that nonalcohol related metabolic pathways would be differentiated in the course of selection. Subsequent work aimed at elucidation of the mechanism or mechanisms underlying the disparate responses of these lines to various drugs is underway.

\section{REFERENCE NOTE}

1. Shapiro, N. R., \& Riley, E. P. Avoidance behavior in rats selectively bred for differential alcohol sensitivity. Manuscript submitted for publication, 1979.

\section{REFERENCES}

Belknap, J. K., Waddingham, S., \& Ondruse K, G. Barbiturate dependence in mice induced by a single short term oral procedure. Physiological Psychology, 1973, 1, 394-396.

Damuanovich, R. P., \& MacInnes, J. W. Factors involved in ethanol narcosis: Analysis in three inbred strains. Life Sciences, $1973,13,55-65$. 
Fog, R. Behavioural effects in rats of morphine and amphetamine and of a combination of the two drugs. Psychopharmacology, 1970, 16, 305-312.

Kakihana, R., Brown, D. R., McClearn, G. E., \& Tabershaw, I. R. Brain sensitivity to alcohol in inbred mouse strains. Science, 1966, 154, 1574-1575.

Lester, D., Lin, G., Anandam, N., Riley, E. P., Worsham, E. D., \& FreED, E. X. Selective breeding of rats for differences in reactivity to alcohol: An approach to an animal model of alcoholism. IV. Some behavioral and chemical measures. In R. G. Thurman, J. C. Williamson, H. Drotte, \& B. Chance (Eds.), Alcohol and aldehyde metabolizing systems (Vol. III). New York: Academic Press, 1977.

Meier, G. W., Hatfield, J. L., \& Foshee, D. P. Genetic and behavioral aspects of pharmacologically induced arousal. Psychopharmacology, 1963, 4, 81-90.

Moisset, B. Genetic analysis of the behavioral response to damphetamine in mice. Psychopharmacology, 1977, 53, 263-267.

Moisset, B., \& WeLCH, B. L. Effects of d-amphetamine upon open field behavior in two inbred strains of mice. Experientia, 1973, 29, 625-626.

Riley, E. P., Freed, E. X., \& Lester, D. Selective breeding of rats for differences in reactivity to alcohol: An approach to an animal model of alcoholism. I. General procedures. Journal of Studies on Alcohol, 1976, 37, 1535-1547.

RILEY, E. P., \& Lochry, E. A. Effects of initial tolerance on acquired tolerance to alcohol in two selectively bred rat strains. Drug and Alcohol Dependence, 1977, 2, 485-494.
Riley, E. P., Lochry, E. A., \& Freed, E. X. Differential tolerance to pentobarbitol in rats bred for differences in alcohol sensitivity. Psychopharmacology, 1978, 58, 167-170.

Riley, E. P., Shapiro, N. R., Lochry, E. A. Hypnotic susceptibility to various depressants in in rats selected for differential ethanol sensitivity. Psychopharmacology, 1979, 60, 311-312.

Riley, E. P., Worsham, E. D., Lester, D., \& Freed, E. X. Selective breeding of rats for differences in reactivity to alcohol: An approach to an animal model of alcoholism. II. Behavioral measures. Journal of Studies on Alcohol, 1977, 38, 1705-1717.

Winer, B. J . Statistical principles in experimental design. New York: McGraw-Hill, 1971.

Worsham, E. D., \& FreED, E. X. Dose-response effects of alcohol upon rat strains bred for differences in reactivity to alcohol. Pharmacology, Biochemistry and Behavior, 1977, 7, 421-424.

Worsham, E. D., Freed, E. X., Lochry, E. A., \& Riley, E. P. Generality of differential sensitivity to alcohol in selectively bred rats. Physiological Psychology, 1977, 5, 429-432.

Worsham, E. D., Riley, E. P., Anandam, N., Lister, P., FreED, E. X., \& LESTER, D. Selective breeding of rats for differences in reactivity to alcohol: An approach to an animal model of alcoholism. III. Some physical and behavioral measures. In M. M. Gross (Ed.), Alcohol intoxication and withdrawal: Experimental studies (Vol. IIIa). New York: Plenum Press, 1977.

(Received for publication April 9, 1979; revision accepted August 14, 1979.) 